\title{
Design Requirements for Mobile TV
}

\author{
Hendrik Knoche \\ University College London \\ Gower St \\ London, WC1E 6BT, UK \\ +44207679 3642 \\ h.knoche@cs.ucl.ac.uk
}

\author{
John D. McCarthy \\ University College London \\ Gower St \\ London, WC1E 6BT, UK \\ +442076793644 \\ j.mccarthy@cs.ucl.ac.uk
}

\begin{abstract}
- In this paper we examine the interaction and delivery requirements for Mobile TV. By comparing the features of traditional TV with current Mobile TV services we outline the design requirements for a future Mobile TV interface. The proposed design is implemented on an iPAQ and evaluated with end-users in a field trial. Preliminary results of the user study suggest that use of the interface is intuitive and successful in giving a TV-like experience. As a secondary study we investigate the bandwidth requirements for different types of TV content on a $3 \mathrm{G}$ phone and a PDA. The results reveal marked differences in bandwidth requirements for different content types. The findings are discussed in the context of future Mobile TV services.
\end{abstract}

\section{Categories and Subject Descriptors}

H.5.2 [Information Interfaces and Presentations]: User Interfaces - evaluation/methodology, prototyping, user centered design, interaction styles

\section{General Terms}

Design, Experimentation, Human Factors

\section{Keywords}

Mobile, TV, SDMB, Satellite, Bandwidth

\section{INTRODUCTION}

This paper explains the design considerations for Mobile TV, accessed through mobile phones or other small screen devices (PDA's). The proposed design is the prototype for a service provided by the Satellite Digital Multimedia Broadcast (SDMB) system under development in the European IST project MAESTRO.

SDMB will deliver multimedia services to European mobile users in a cost efficient manner [1]. The distribution of content will be achieved by integrating satellite broadcast capacities with existing terrestrial $3 \mathrm{G}$ and beyond $3 \mathrm{G}$ infrastructures (e.g. DVB-H). The SDMB service is received

Permission to make digital or hard copies of all or part of this work for personal or classroom use is granted without fee provided that copies are not made or distributed for profit or commercial advantage and that copies bear this notice and the full citation on the first page. To copy otherwise, or republish, to post on servers or to redistribute to lists, requires prior specific permission and/or a fee.

MobileHCI'05, September 19-22, 2005, Salzburg, Austria.

Copyright 2005 ACM 1-59593-089-2/05/0009...\$5.00. using a normal $3 \mathrm{G}$ phone antennae and assumes mobile devices with extensive storage and battery capacity. This means that users can consume previously cached content at opportune times as well as watching live streams or receiving information bulletins.

To understand the requirements for this type of service we consider both the interaction and delivery requirements for mobile TV services. Interaction requirements cover the users access, navigation and experience of content. Delivery requirements encompass the encoding, transmission and decoding of content to deliver an acceptable Quality of Service.

The first half of this paper deals with the interaction requirements. In designing these we first consider a number of issues. These include:

1. How and why people watch traditional television.

2. Current use of mobile phones and "TV" services.

3. Types of content people want to see while they are mobile.

In Section 2 we will address each of these issues in turn. In Section 3 we describe the design of the interface based on the requirements derived in Section 2. In Section 4 we present results from a field evaluation of the interface, specifically focused on usage by long commuters. Section 5 introduces the importance of encoding bitrate to delivering an acceptable Quality of Service. Section 6 presents a study of encoding bitrate for different content types on two different mobile devices. Finally, in Section 7, we conclude with a brief discussion of the technical and service implications for Mobile $\mathrm{TV}$ in general.

\section{INTERACTION REQUIREMENTS}

With mobile phones capable of displaying multimedia (AV) content and 3G networks capable of delivery, mobile multimedia consumption has become a reality. To the authors' knowledge, the design rationales of current mobile TV interfaces have not been the subject of scientific discourse.

Existing research has focused on the design and usability issues with full size TV screens, examining issues such as the design of electronic programming guides (EPG) [2] and interactive TV [3].

Little work has been published on effective browsing of digital television content. The effectiveness and efficiency of different video skipping approaches in digital television have been studied in [4]. However, this research also addressed full size television screens and devices with a considerable amount of 
computational power. The tasks were commercial skipping and finding the weather information within a news broadcast. Other studies have examined how users skim video [5] or determine the overall gist of content. [6]. In the following sections we highlight the differences between traditional TV interaction and that currently implemented on mobile devices.

\subsection{Traditional TV Consumption}

People watch television for social and psychological reasons. On the social side people value the time they are together with friends or family and enjoy a communal experience. On the psychological side, mood management is one of the major drivers. If people are bored, they choose excitatory or arousing content, if they are stressed, they prefer relaxing content [7].

Television consumption generally takes place at home and is usually a "lean back" activity, where an audience passively consumes broadcast content. However, remote controls can facilitate channel hopping in an opportunistic search for programmes that satisfy a viewer's moods and mindsets. In [8] it was argued that channel surfing is inherently associated with the act of watching TV. When in a group, however, the decision of what to watch is a contentious one. According to a survey, 9 in 10 families say they regularly disagree over what to watch [9].

The methods to select a program used in traditional TV viewing depend on the time of day. But generally the method used escalates - if nothing of interest is found - to strategies that require more effort on behalf of the user[8]. The ordering of strategies is

1. Channel surfing

2. Wait or search for a program announcement on TV

3. Knowledge of weekly schedules or upcoming programs

4. Paper-based or onscreen guides

Viewing also has varying levels of commitment. There is a higher level of commitment with favourite shows, or programs that people plan for ahead of time, and shows that viewers have to pay for on a per-use basis. During these shows, people are less open to outside interruptions. Low commitment viewing on the other hand exhibits more channel hopping, competes with many other demands and activities within the home, and might be just a background activity.

In terms of interaction and user experience, traditional TV has some very specific features. For the context of our work we consider the following to be the defining characteristics:

- Instant On - Once you turn on the TV you receive content of a channel immediately.

- Continuous - Channels show content all the time. Once one programme ends another one begins.

- Seamless Switch - The switch to a parallel channel is more or less instantaneous

- $\quad$ Easy Switch - The cost of switching to a different channel is low especially with a remote control that overcomes seat inertia

- Graceful Transitions - The transitions from one program to the next program are smooth as broadcasters try to keep their viewers

- $\quad$ Reliable service - After its initial setup a breakdown of TV is very uncommon.
It is interesting to contrast these features with the interaction style and user experience of existing mobile multimedia services.

\subsection{Mobile Phones}

The use of mobile phones evolves around the three general user areas of home, work, and public [10]. The mobile phone has transcended its original role as a means of communication by now serving a multitude of purposes. It has brought about new social norms and behaviours, e.g. emergent phenomena in young adults' socializing patterns.

A mobile phone is not only a highly personal tool that many feel dependent on, but also many aspects of being with and being apart from others evolves around it. Its main usage is to stay in touch with friends and family and to synchronize with them in and across space and time. The perceived threats to this need are the inherent high cost, imperfect coverage, and short battery life [11]. To many, usage of mobile phones does not necessarily imply being on the move but constitutes a means of communication that is ready at hand. Previous research has shown that the majority of people who are interested in mobile $\mathrm{TV}$ are willing to make compromises on the size of the screen for the sake of simplicity and portability [11]. However, many people preferred a separate device that would have a bigger screen and not consume power they might need for communication.

\subsection{Current Mobile TV services}

There are two service types that use the term mobile TV. The first is a download-oriented service through which videos are accessed via galleries that include a thumbnail and a title describing the content (as depicted in Figure 1).

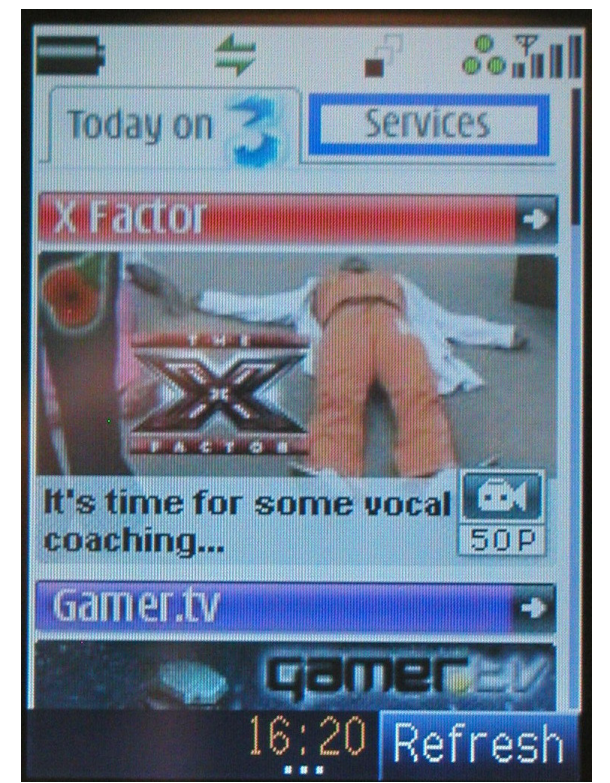

Figure 1: Current Mobile TV service - gallery based

The typical question that arises after a clip has finished playing is: What next? Whereas traditional impromptu television choices are based on the content, and the point of entry into the content - the consumption of video on demand requires more interaction and decision-making. Unlike traditional TV there is 
no opportunity for easy channel hopping, and the naming of items in a gallery or programme guide exerts a strong influence on user choice. Thus, if an item is renamed with certain 'key' words, e.g., forbidden, explicit, illicit, and sex, the download frequency will be dramatically increased [12].

A list of the features of current services lies in stark contrast to those of traditional TV:

- Delayed On - A programme must be chosen from a gallery before it is shown.

- Discontinuous - Once a programme has finished nothing else is shown.

- No Transitions - There are no transitions from one program to the next. Once a clip has played. That's it.

- Difficult Switch - The cost of switching to a different channel is high, as galleries or programme guides have to be traversed and decisions must be made each time.

- Interrupted Switch - The switch to a different channel or programme is delayed by the decision making process.

- Geographical reliability - Depending on the position of the device there might be no access to the service.

The second service type (e.g. DVB-H) is based on the parallel terrestrial broadcast of streamed channels. This service, e.g. mobiTV [13] has a similar behaviour to standard TV in many respects. For example, after switch on and a buffering period, MobiTV automatically starts playing a default channel.

Switches to other channels can be done either through a shortcut plus the channel number or by using the up and down button. Switches to another channel are not instantaneous, however, but take from approximately five to fifteen seconds; similar to the delay of the initial channel after the application is launched. If there is no coverage in an area one cannot watch mobiTV.. Nor can MobiTV be paused if a situation demands the users' full attention and. In addition, due to the streamed broadcast, users might turn on mobiTV at times when the programs in which they are interested in are not on.

In short the comparison to standard TV looks like this:

- Slightly delayed On - The duration of tuning into a channel depends on the encoding of the channel content and the capabilities of the device.

- Transitions - Like traditional TV.

- Slightly delayed switch - Same as for "Slightly delayed on"

- $\quad$ Easy Switch - On phones the number keys can be used as they can on standard remote or channel. Many mobile telephone layouts also sport an up and down key similar to many remote controls.

- Geographical reliability - During movement the service might intermittently become available. Depending on the position of the device there might be no access to the service at all.

What should be clear from this analysis is that current TV like services on mobile phones offer a different experience to that of traditional TV and will not deliver a true TV anytime, anywhere experience which users seek in their mobile phones [11].

\subsection{Content and Service Requirements}

To understand content requirements for Mobile TV we conducted focus groups in three European countries - the UK, France and Greece. In all, 160 people were asked about the problems they were experiencing and the needs they had with respect to their current phones and services as well as their expectations of future mobile multimedia services. The wide range of demographics across the groups included teenagers, young professionals and older people with families. Full details of the focus group findings are presented in [11].

In terms of interaction design a number of findings are of relevance: (i) the most popular content was news, (ii) viewing was likely to be transient and low commitment, (iii) people were worried about getting too absorbed and being distracted from other tasks and finally (iv) it was unlikely that mobile TV would be watched in a group.

Being mobile consists of activity spurts that are interleaved with periods of dead or unstructured time. In light of this, we anticipate low commitment viewing to be the more dominant consumption pattern on a mobile device because of the strong influence of the environment, the fact that attention might compete with other, possibly more important needs while being mobile, and last but not least the functional overlap with users' means of communication. Previous research has shown that peoples' average [13] usage of mobile TV during these periods is less than ten minutes long. This has ramifications both on the type of content as well as the way that people consume it. Longer programs will be more appealing to people that experience extensive dead times, such as long commuters [11].

People are worried about being absorbed by multimedia content, which requires their visual attention and is constantly progressing at its own pace. They fear increased risks of accidents or lapses (e.g. missing train stops) [11]. Furthermore, many people are wary of the effect their mobile phone usage, i.e. talking aloud, has on others in public spaces. For these users, multimedia consumption requires the use of headphones, which might further immerse them.

Despite marketing efforts to the contrary, focus groups suggest that watching TV on a phone will often be a serial solitary activity. This is not only based on the fact that current screens and their viewing angles are not conducive to these kinds of endeavours, as viewers have to move uncomfortably close together [13], but also on the more fundamental fact that the mobile phone is a personal device. Its use is tied to its owner's needs to stay in touch and be in control, which requires exclusive control over the handset.

\section{DESIGN AND IMPLEMENTATION}

\subsection{Interaction Design}

Having examined the interaction with traditional TV and current multimedia services it is clear that there are many differences. For the majority of users, the window of opportunity for mobile TV consumption is short and the interface should allow users to select the content in a $T V$ anytime fashion [14], i.e., from the beginning and without technically induced delays or a lengthy task of accessing the content.

If all content is streamed, then these requirements will not be fulfilled. The user is more likely to tune-in to the middle of a program and the service may be temporarily unavailable because 
of reception difficulties. For both these reasons it is very important that content is downloaded and cached on the device so that there is always something available to watch - at anytime.

With this in mind we focused our design on emulating the experience of traditional TV with features that facilitate viewing in a mobile context.

One of the first design decisions was to remove the program guide or gallery as the primary means of accessing content. As described earlier, putting the guide between the user and the content significantly changes the nature of the TV experience. In addition, the cost or cognitive overhead of having to actively choose every program that is watched is likely to be a major inhibitor to consumption and is not conducive to impromptu viewing.

Similar observations have been made in studies of digital television. For example, Eronen and Vuorimaa found that users who were interested in watching television were not interested in interacting with an EPG or interactive television. The authors emphasized that digital television should maintain the familiar living room TV experience [15].

To maintain this experience for mobile devices, we adopted a design for the mobile consumption of TV channels that was based on content items as originally conceived by [16], which make up part of a program. As with traditional TV, programs are serially aligned in channels. In our design Channels are accessed through interface "tabs" as depicted in Figure 2.

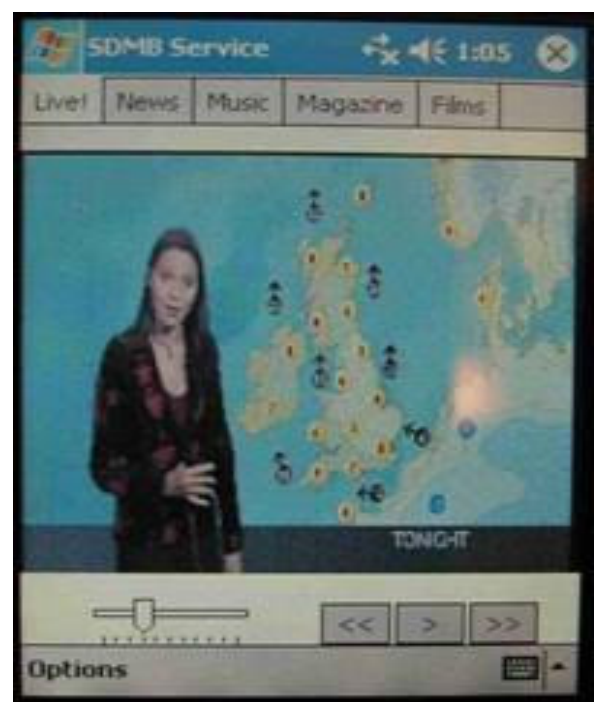

Figure 2: Image of the mobile TV interface. Channel tabs on top, skip forward and back buttons on the lower right with the Pause/Play button in the middle and the audio control slider is located to the left of these buttons.

These channels can offer access to streamed broadcasts, downloaded programs or a combination of the two. To create the familiar TV feel we adopted four key interaction principles.

\subsubsection{ON}

Mobile TV should behave like an ordinary TV. When turned on, it starts playing content that may or may not be of interest to the user. If the user does not interact with the mobile TV software it will continue showing content. If there is no new content it should loop back to the first program again. The only exception to this is if a low battery threshold is reached such that the user's communication needs would be compromised.

We are not advocating the abolishment of an electronic program guide (EPG), which constitutes a valuable instrument in content navigation [14] and will be added in future versions of this interface. But we are not convinced that an EPG is a good entry point into a mobile TV service.

\subsubsection{HOP}

The envisaged use of mobile television is usually dead or unstructured time. Therefore, we expect users to exhibit low commitment viewing unless their expected time frame for viewing is much longer than ten minutes e.g. for long commuters. If the user is not interested in a channel or program he can switch or hop quickly and easily to a different channel by a single press of a button or by selecting its channel tab on the screen.

\subsubsection{SKIP}

In other studies users had voiced their desire for indexed programs that would allow them to skip to interesting parts or scenes [14]. We include this functionality. If the user is not interested in a content item but wants to keep watching other items within the same program he can skip to the next content item. There is also the opportunity to move backwards and forwards between whole programs.

With these two methods of surfing the interface allows for more habit formation [17], in contrast to lists displaying video clips, which differ everyday or possibly every time a user browses them. If the user wants to engage in more complicated navigational methods, or search for content, the user will be able to use an EPG in future versions of this interface.

\subsubsection{PAUSE}

Time-shifted viewing, as promoted in hard disk based video recorders, will be possible by means of a pause button. If for any reason, e.g., to get on a bus, the user needs to pause the program at its current position the user can press the pause button. For incoming calls the presentation of the content should be automatically paused and the user confronted with the question of whether to accept or reject the call.

\subsection{Content Mix and Types}

The channels chosen for the interface reflect the relative importance given to different content types by users in focus groups [11]. As the most demanded service, News is given a dedicated channel, and the latest News broadcast is played as soon as the device is turned in. There is also a dedicated channel for live or pay per view broadcasts, and one for music or MTV. All other programs are included in a generic magazine channel the contents of which can be specified by the user. Finally, we included a channel for short films. Advertisements, which could also be skipped, were used, however, at a much lower frequency than in real TV.

As the majority of TV programmes are produced well in advance of their broadcast, the delivery of content in advance is straightforward. An important exception to this is News, which must be as recent as possible. However, even with News 
coverage a broadcast from 1-2 hours ago is still considered "recent". Because of this perception, it is possible that even cached News broadcasts are a viable service. To examine this in more detail we cached a News broadcast from earlier in the day on the device for the evaluation. In total 2.5 hours of content were stored and available for immediate viewing.

\section{INTERACTION EVALUATION}

\subsection{Users}

From the focus groups it was clear that the people with the most time and opportunity to watch mobile TV would be long commuters. As these people had plenty of dead time during which they usually read books, papers or listen to music we were interested in how mobile TV stacked up against these competing activities. Consequently we advertised for volunteers on the 17:15 commuter train between London Liverpool Street and the rural town of Southminster. As the crow flies Southminster is around 50miles $(110 \mathrm{~km})$ outside London and the total journey time is around 59 minutes. This train only has few stops that are quite long such that missing a stop is far less likely than on inner-city subway trains.

As a case study for the SDMB service the long commuters are particular interesting as these users move to and from rural locations. For seamless delivery of TV content to these users a satellite system is essential as complete coverage by terrestrial transmitters in rural areas (e.g. DVB-H) is unlikely to be economically viable.

We had four volunteers for the evaluation, these were, Gus, a 46-year-old man and his 11-year-old daughter. The daughter commuted daily to a specialist stage school within the city. The other volunteers were, Carol a 58-year-old woman and Helen a 43-year-old.

\subsection{Protocol}

A relatively simple protocol was adopted for the evaluation. We gave each of the volunteers an iPAQ device and showed them how to turn it on. The iPAQ was equipped with a pair of headsets so the participants would not worry about annoying fellow commuters. We then told them they where free to use the device however they wished for the next 30 minutes after which time we would ask them a number of questions. No help or guidance on how to use the interface was given but we told people that we would help them if they got stuck.

The questions asked after they had used the device where the following:

1. Did you enjoy it?

2. Was it similar to watching normal TV?

3. Did you find it easy to use?

4. Did you encounter any problems?

5. Were there any features you would add?

6. Were you happy with the range of programs?

7. Did you tend to channel hop?

8. Did the adverts bother you?

9. Would you pay for such a service?

10. Would you prefer to watch "live" TV or stored content?

11. What would do you normally do on the train?

12. Would you watch mobile TV instead?

Answers to these questions were recorded using a DV camera.

\subsection{Results}

As the answers to some of the questions were quite long, a full presentation of the transcript is not presented here. However to give an indication of the general attitudes we present a summary of whether people responded positively or negatively in Table 1 below. A "YES" response is shaded in light grey, a "NO" is shaded in black.

As can be seen in the table there is a surprising degree of consistency across the four people tested. Everyone enjoyed it, found it easy to use and said it was similar to watching normal TV. Only the first person tested encountered any technical problems, and this was due to an unrelated iPAQ power saving feature that turned the screen off after 5 minutes. This feature was disabled in subsequent tests. The two features people wanted to add where an ability to change the picture quality and the addition of a program guide.

\begin{tabular}{|l|l|l|l|l|}
\hline Did you enjoy it? & & & & \\
\hline Was it similar to watching normal TV? & & & & \\
\hline Did you find it easy to use? & & & & \\
\hline Did you encounter any problems? & & & \\
\hline $\begin{array}{l}\text { Were there any features you would } \\
\text { add? }\end{array}$ & & & \\
\hline $\begin{array}{l}\text { Were you happy with the range of } \\
\text { programs? }\end{array}$ & & & \\
\hline Did you tend to channel hop? & & & & \\
\hline Did the adverts bother you? & & & \\
\hline Would you pay for such a service? & & & & \\
\hline Would you prefer to watch "live" TV? & & & & \\
\hline Do you normally read on the train? & & & & \\
\hline Would you watch mobile TV instead? & & & & \\
\hline
\end{tabular}

Table 1: User responses in the evaluation. $=$ =yes, $\square=$ no

Everyone was willing to pay for such a service and said they would watch mobile TV instead of reading on the train. Only one person was bothered by adverts in the content..

Three of the four people said they would prefer to get live $\mathrm{TV}$ - yet when we quizzed them further, it was clear that what they wanted was programs that were in the normal TV schedule. How they were actually delivered was not important. For News coverage however, there is a strong case for live or nearly live delivery. The news broadcast people saw in the evaluation was around 5 hours old (midday news) and everyone commented that this was not recent enough.

\subsection{Discussion}

The interaction design we adopted for Mobile TV draws from previous research on television watching behaviour and psychology, mobile phone usage, and mobile multimedia consumption. The prototype ports many of the traditional TV characteristics to the mobile device. A service that starts presenting content immediately on start up, lets users hop quickly though the channels, skip boring parts and pause the content is an essential approach to watching audiovisual content while on the move.

Initial evaluation of the interface is promising and suggests we were successful in designing a TV experience. Further 
evaluation and design iterations will be conducted to implement the additional features users demand and examine the delay tolerance for News broadcasts. In the following section we focus on the bandwidth requirements for Mobile TV.

\section{MOBILE TV DELIVERY}

\subsection{Service Scenarios and Content Types}

Within the Mobile TV concept there are a number of different scenarios. These range from Live TV, to Push and Store services or even transfer from a users' Personal Video Recorder (PVR) in the home. Where TV is received directly on the mobile, there are again a number of possibilities including reception via terrestrial (e.g. DVB-H) or satellite (e.g. SDMB) networks. Whereas DVB-H is intended for the broadcast of many live channels in parallel, SDMB focuses more on push and store services. The main technological difference, however, is the area of coverage. By using satellite broadcasting, SDMB gives good coverage in rural areas, something which is unlikely to be economically viable using terrestrial DVB-H broadcasting.

While the convergence of Media (TV) and Mobile environments seems inevitable in the long run, little is known about the technical requirements to deliver an acceptable Quality of Service (QoS) or Quality of Experience (QoE) [18]. Existing research has tended to focus on Sports as a service [19], [20], [21]. As little is known of the requirements for other types of services, we conducted an evaluation study. A primary focus of the study was the bitrate requirements for different service types.

\subsection{Encoding Bitrate}

The encoding bitrate is the predominant factor that influences video and audio quality on the user equipment, and for lower encoding bitrates, more content can be transmitted to the receivers. For Mobile TV services it is important to distinguish two different relationships between encoding bitrate and the available transmission rate. With real-time streaming of live or pre-recorded material, the encoding bitrate cannot be

\begin{tabular}{|l|l|}
\hline Service & Content \\
\hline Movie & Kill Bill 2 movie trailer \\
\hline Music clip & Beastie Boys MTV music video clip \\
\hline Football & Goal highlights: Man. U vs. Arsenal \\
\hline News & CBSNews news clip \\
\hline Weather & BBC UK weather update \\
\hline
\end{tabular}

Table 3: TV content clips of the five service types.

higher than the available transmission rate. In contrast, with Push and Store services the video content can be encoded at higher bitrates than the transmission bitrate as the delay requirements for delivery are relaxed. It is possible therefore that material encoded at $1 \mathrm{Mbps}$ could be delivered over a $256 \mathrm{kbps}$ link for viewing at some later date. (e.g. the next day).

Different classes of content or services will also have different ranges of bandwidth requirements. As described by Apteker et al. the bandwidth requirements for TV are a function of the temporal nature of the data and the importance of the audio and visual channels in understanding the message [21].
Generally, content with a high degree of change, for example, a moving background due to camera pans (Highly Temporal Data), requires more bandwidth. Thus, highlights from a football match would require significantly more bandwidth than highlights from a snooker match.

To examine these issues further we conducted an evaluation of bandwidth requirements for 5 different content types with a small sample of users.

\section{BANDWIDTH EVALUATION}

\subsection{Users}

We randomly selected a group of 20 users from a University Subject Pool to take part in the study. The mean age of the participants was 26 . Each participant was paid approx $€ 15$ for participation.

\subsection{Protocol}

To investigate bandwidth requirements for different service classes we adapt the measure of Acceptability used by [22] to measure the percentage of the target group that find a given level of service acceptable. We also asked users to rate the acceptability of video quality on two different devices - an iPAQ a handheld computer (PDA) and an NEC 3G phone. The devices had different resolutions and supported different video codecs. The Windows based iPAQ supports the WMV codec whereas the Symbian based NEC phone supports the 3GPP MPEG 4 standard. The codecs, resolutions and range of bitrates tested are presented in Table 2.

\begin{tabular}{|l|l|l|l|l|}
\hline & & & \multicolumn{2}{|c|}{$\begin{array}{r}\text { Bitrate Range } \\
\text { (kbps) }\end{array}$} \\
\hline Device & Codec & Resolution & Total & Video \\
\hline $\begin{array}{l}\text { IPAQ } \\
2210\end{array}$ & WMV & $320 \times 240$ & $64-512$ & $52-448$ \\
\hline $\begin{array}{l}3 \mathrm{G} \\
\text { PHONE }\end{array}$ & $\begin{array}{l}\text { 3GPP } \\
\text { MPEG4 }\end{array}$ & $176 \times 144$ & $28-256$ & $16-243$ \\
\hline
\end{tabular}

Table 2: Encoding parameters for the two devices

Representative TV content was chosen to correspond to 5 different services. Each clip was 15 seconds in length which is similar to the duration of clips used in experiments that comply with ITU subjective testing standards $[23 ; 24]$. Details of the content are presented in Table 3.

Each user was shown each of the clips at a range of different bandwidths and asked to indicate whether they found the quality acceptable or unacceptable.

\subsection{Results}

The mean acceptability figures reported in the results also correspond to the proportion of our sample that rated a given bandwidth as acceptable in terms of video or audio quality. Using this measure we found very different bandwidth thresholds for different types of services at a resolution of $320 \mathrm{x}$ 240. The most demanding type of content was the football coverage (See Figure 3). Even at $448 \mathrm{kbps}$ this service did not achieve $90 \%$ acceptability but reached a plateau for bitrates of $332 \mathrm{kbps}$ and greater at $84 \%$. This might be due to the resolution and/or screen size and represent an unsurpassable value for this device. In stark contrast, News and Weather delivered an 
acceptable service to $90 \%$ of people at a bandwidth of just $112 \mathrm{kbps}$. However, to reach a similar acceptability the Music and Movie content needs to be coded at twice the former rate (224kbps).

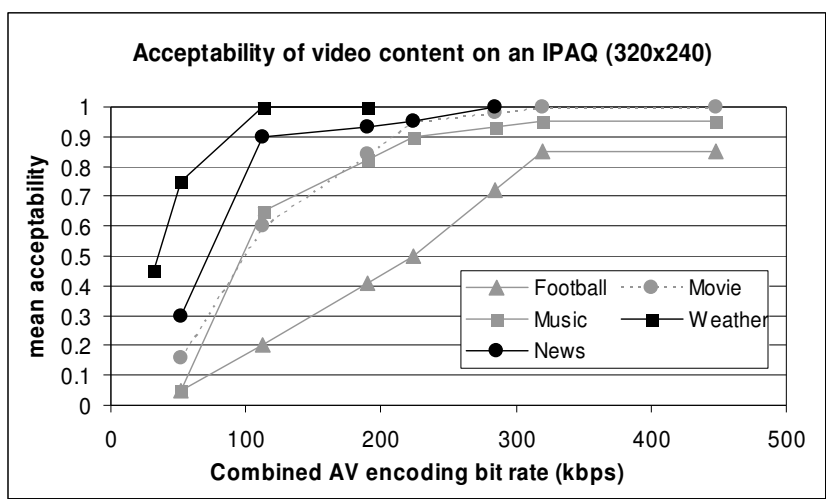

Figure 3: Content Requirements on an iPAQ.

A very different pattern of results was produced for content encoded using 3GPP MPEG 4 and viewed on the $3 \mathrm{G}$ phone at $176 \times 144$ (See Figure 4). At this resolution Movie content required less than half the bitrate $(111 \mathrm{kbps})$ than on the IPAQ to be acceptable to $90 \%$ of the participants. All other content categories also achieved high acceptability at $111 \mathrm{kbps}$. The football coverage reached about $90 \%$ acceptability at $243 \mathrm{kbps}$, in contrast to its poor acceptability (ca. $60 \%$ interpolated) at a comparable bitrate on the IPAQ.

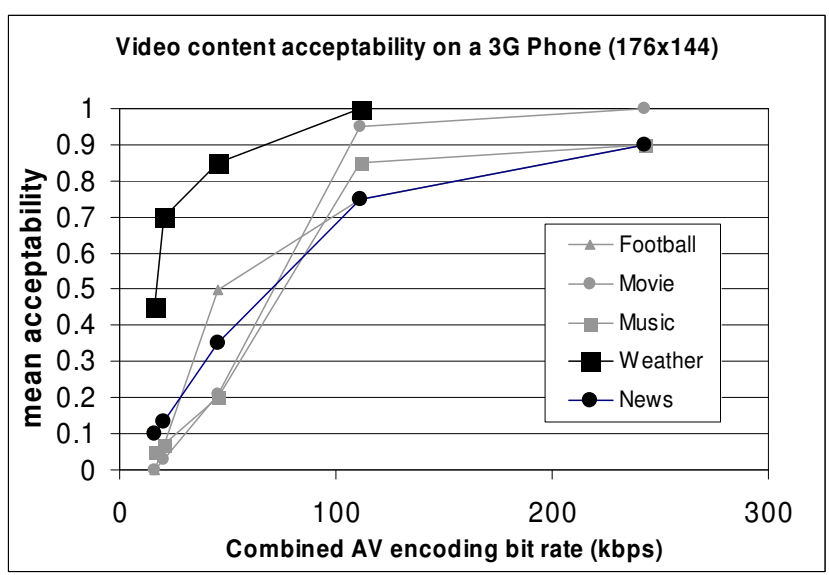

Figure 4: Content requirements on a $3 G$ phone.

This is quite interesting as it suggests that people would watch football encoded at $243 \mathrm{kbps}$ on a smaller screen but not on the larger iPAQ. However, with the current sample size it could also be an outlier. Weather was again acceptable to almost $100 \%$ of users at $111 \mathrm{kbps}$. This can be explained by the absence of motion in the picture, in fact the background of the video depicting the weather map is static during the duration of the clip. For all other types of content acceptability drops off sharply as the bandwidth drops below $111 \mathrm{kbps}$. On the higher resolution iPAQ this drop off had occurred at much higher bitrates.

\subsection{Discussion}

The results of the evaluation illustrate the substantially different bandwidth requirements for different types of service with News and Weather being the most bandwidth efficient. However, we note that there are a number of other factors that may have contributed to users' perception. For example, the audio bitrate also varied across different clips so it is possible that low quality audio may have impacted on users rating of video. Another factor is the quality of the LCD display on the two devices, with the LCD on the phone offering much lower contrast on than on the iPAQ. For these reasons comparisons across devices are probably unfair. Nevertheless the results clearly indicate that on each device, at any particular coding level, there are clear differences in acceptability between different types of content and highlight the need for further research.

\section{CONCLUSIONS}

\subsection{Methodological}

The use of prototyping and service simulation to evaluate the interaction and service requirements gave valuable feedback to guide future design iterations. Despite the fact that no program guide was provided users found the interaction enjoyable, compelling and similar to normal TV. Giving users extended experience with the service and device in a realistic usage setting also provided valuable information on the delay requirements for delivering popular content types such as News. Future work will map the prototype interaction style onto the specific requirements of phone based interaction and consider more interactive services associated with particular TV content (e.g. RSS feeds, ring tones and games).

For the bandwidth evaluation the measure of service acceptability produced clear psychophysical functions relating physical quality to perceived acceptability. We would recommend the using of such an approach to evaluate bandwidth requirements instead of the widely used alternatives such as ITU methodologies $[22 ; 23]$.

\subsection{Substantive}

The initial findings from the interaction evaluation lend support to the four interaction principles we established for Mobile TV. Given that use of Mobile TV is likely to be impromptu and low commitment we would strongly advocate an interaction paradigm that minimizes the barriers to entry and exploration of content.

Of course, these conclusions have consequences on other aspects of Mobile TV usage such as charging or payment interaction. With existing services, charging is usually imposed at the level of the TV clip. This is problematic in the current design, as it would severely interrupt the flow as defined in [25] of content exploration and experience. Our current recommendation is to charge for a selection or bouquet of content instead. Thus, users would receive a bouquet or play list of TV on a daily basis for a fixed price per month. The reactions to such charging schemes will be evaluated in a future study.

In terms of bandwidth requirements our initial findings suggest that it may be possible to identify different content classes with different bandwidth requirements. Here again however commercial considerations come into play. For example, although we found that football demanded a very high bandwidth, there exist services delivering football coverage at much lower bandwidths. This is achieved by specially editing the material so that it can be delivered over very low bandwidths. With football coverage this means excluding certain shot types (long/distant shot from the stand) and 
cropping shots to focus in the information of most importance to the user [22]. Editing in this way however, costs both time and money. If material is to be delivered live or tailor-made editing is not economical then the bandwidth requirements we have identified should hold.

\subsection{Future work}

We are planning to make the channel hopping, program skipping and pausing available through buttons, such that the interface can be operated single-handedly by thumb as advocated in [26].

Since people use their TV to temporally structure their lives [27] we also plan to maintain some notion of real time in the cached content. A program that is usually broadcast at a certain time would be the default choice when switching to that program on mobile TV during that time window.

As part of future work we are examining how best to maintain perceived Quality of Service for the Satellite Mobile TV service. Generally speaking the approach is to store as much material locally as is feasible and update this on a regular basis. Thus, even if users have no real reception (deep indoors, underground) there will still be content for them to watch and explore.Our next step is to understand how different ways of delivering the content to the terminal ultimately affect the perceived quality of the Mobile TV service. We plan to explore this with clips of realistic length, e.g. 2-5 minutes to ensure the validity of the obtained results.

\section{ACKNOWLEDGMENTS}

Our thanks to Odyssey software, inc. for the provision of CFCOM, the EU for funding the Maestro IST project and Professor Angela Sasse for her guidance with this research.

\section{REFERENCES}

[1] Narenthiran, K., Karaliopoulos, M., Tafazolli, R., Evans, B. G., Vincent, P., Selier, C. et al. S-DMB System Architecture and the MODIS DEMO in IST Mobile and Wireless Communications Summit 2003, 2003

[2] Concejero, P., Gil, S., Ramos, R., Collado, J. A., Castellanos, M. A. Usability testing on an electronic programme guide and interactive in Proc.of Human Factors in Telecommunication, 1999

[3] Chorianopoulos, K. Virtual Television Channels Conceptual Model, User Interface Design and Affective Usability Evaluation. PhD Athens University of Economics and Business, 2004

[4] Drucker, P., Glatzer, A., De Mar, S., Wong, C. SmartSkip: Consumer level browsing and skipping of digital video content in Proceedings of the SIGCHI conference on Human factors in computing systems: Changing our world, changing ourselves, p. 219-26, New York, NY, USA: ACM Press, 2004

[5] Chistel, M., Smith, M., Taylor, C., Winkler, D. Evolving video skims into useful multimedia abstractions. in Proceedings of CHI '98, ACM Press, 2004

[6] Toney, T., Vegh, S., Marchionini, G., Shneiderman, B. An Exploratory Study of Video Browsing User Interface
Designs and Research Methodologies: Effectiveness in Information Seeking Tasks. in Proceedings of the 62nd ASIS Annual Meeting., 1999

[7] Zillman, D. Mood Management: Using Entertainment to Full Advantage. In L.Donohew, H. E. Sypher, \& E. T. Higgins (Eds.), Communication, Social Cognition, and Affect (, p. 147-72). Hillsdale: Erlbaum, 1988

[8] Taylor, A. \& Harper, R. Switching on to switch off: An analysis of routine $T V$ watching habits and their implications for electronic programme guide design usableiTV, 1 (3), p. 7-13, 2002.

[9] Bruckland, H. Survey reveals Brits choose Telly over Turkey! Sky Digital Publicity. 2002.

[10] Edelmann, J. \& Koivuniemi, J. Future development of mobile services and applications examined through the real options approach Telektronikk, 2, p. 48-57, 2004.

[11] Knoche, H. \& McCarthy, J. Mobile Users' Needs and Expectations of Future Multimedia Services in Proceedings of the WWRF12, 2004

[12] Buck, B. The worlds smallest film festival - Onadime. 2004.

[13] Sprint, MobiTV., http://www.mobitv.com/. 2004

[14] Södergård, C. Mobile television - technology and user experiences Report on the Mobile-TV project (Rep. No. P506) VTT Information Technology, 2003.

[15] Eronen, L. \& Vuorimaa, P. User preferences and designers' views on what would suit the user. in Proceedings of the Working Conference on Advanced Visual Interfaces, ACM Press, 2000

[16] Negroponte, N. Being Digital New York: Vintage, 1996

[17] Raskin, J. The Humane Interface Addison-Wesley, 2000

[18] Jain, R. Quality of Experience IEEE Multimedia, 11, p. 956, 2004.

[19] Wikstrand, G. \& Sun, J. Determining utility functions for streaming low bitrate football video in Proc.of IMSA, 2004

[20] McCarthy, J., Sasse, M. A., Miras, D. Evaluating Mobile Video Quality in Proc.of IST Mobile conference, 2004

[21] Apteker, R. T., Fisher, A. A., Kisimov, V. S., Neishlos, H. Distributed multimedia: user perception and dynamic QoS in Proceedings of SPIE, p. 226-34, 1994

[22] McCarthy, J., Sasse, M. A., Miras, D. Sharp or smooth? Comparing the effects of quantization vs. frame rate for streamed video in Proc.CHI, p. 535-42, 2004

[23] ITU-R Methodology for the subjective assessment of the quality of television pictures. (Rep. No. BT.500-7), 2004.

[24] ITU-T P.800.Methods for subjective determination of transmission quality (Rep. No. P.800), 2004.

[25] Csikszentmihalyi, M. Flow: The psychology of optimal experience New York: HarperCollins, 1990

[26] Eronen, L. \& Vuorimaa, P. User Interfaces for Digital Television: a Navigator Case Study in Proceedings of the Working Conference on Advanced Visual Interfaces AVI 2000, p. 276-9, New York, NY, USA: ACM Press, 2000

[27] Gauntlett, D. \& Hill, A. TV Living: Television, Culture and Everyday Life. Routledge, 1999 